\title{
Finding a way forward in the
}

community
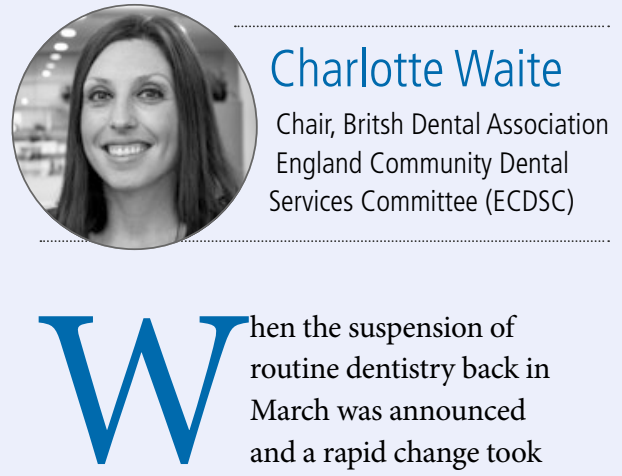

place in the delivery of dental care, from the perspective of community dental teams, these changes came about very quickly. Our teams cancelled clinics, general anaesthetic procedures, and domiciliary visits while reassuring our patients their parents and carers, that we would still be available to provide support and advice. Some community dental team members were re-deployed and others started to work towards establishing the Urgent Dental Care (UDC) Hubs.

I have always been proud to be a community dentist but during this period the compassion, drive and agility of our teams cannot be underestimated. Whether it is locally, regionally or nationally, community dental teams have worked together, shared knowledge and learning, whilst striving for the best, for our teams and patients. People have worked tirelessly to adapt standard operating procedures, to train teams in new ways of working, whilst supporting each other and sharing expertise. This has taken place not only within the CDS but across primary and secondary dental care as well. Members of dental teams have supported their colleagues to establish and prepare UDCs, for example those who have assisted with mask fit testing of colleagues, which was a key part in the expansion of the UDC provision.

Many, CDS dentists are providing urgent dental care for not only paediatric and special care dental patients but also people in the vulnerable and shielded categories, be this as level 1 or level 3 triage and additionally as providers of urgent dental care in UDC Hubs. Also, some of the hubs have been identified to provide urgent dental care for COVID positive/symptomatic patients. Domiciliary visits may be an appropriate option in the first instance, for some shielded patients and CDS have been delivering these services as well.

In truth none of us knows what impact the pandemic will have on dentistry in the short, medium and long term. However, I do feel that much of this work will lay the foundations for the months and possibly years to come and I believe that CDS members are well placed to move forward to the next phase of working.

This month's cover feature provides a number of opinions on that next phase. ${ }^{1}$ Undoubtedly, we will have to adapt our services and work in different ways, and reading through the thoughts of those who work in and across the community it brings home the size and scale of the changes we are looking at. These changes will of course affect service delivery and our patients. It seems likely that there will a reduction in aerosol generating procedures (AGPs) for a period of time. One area where this may have a significant impact is comprehensive care under GA, with both paediatric and adult special care general anaesthetic lists potentially affected. As yet, it is not clear whether GA list capacity will be affected but it would seem likely. There may be an expectation for patients to self isolate for a period of time before the GA procedure and this may cause challenges for children, their families and our special care adult patients, with pressure on theatre lists already caused by the cancellation of elective lists generally throughout the NHS, as well as new patients waiting to be assessed. Moving forward, I feel that it is important that ECDSC works to support the CDS in securing adequate access to GA lists for our patients.

New and innovative ways of working have been rapidly developed and I feel strongly that as holistic clinicians we will maximise these to benefit our patients. It may be beneficial to have remote consultations, particularly for those patients in the shielded or vulnerable groups, in relation to COVID-19. As a profession, we have embraced remote consultations including video consultations but perhaps we could go a step further and work with the digital communications sector to develop bespoke solutions for our dental patients. Social distancing may present challenges for the groups of patients seen within the CDS and some of our patients need multiple people to support them during appointments. This is where planning and individual risk assessments will be key, arranging appointments timings carefully and perhaps considering domiciliary appointments for those in higher risk groups.

Our medical and pharmacy colleagues have supported our patients too and I hope that stronger relationships across primary care can be nurtured, presenting an opportunity for closer collaboration. I believe that key to this, will be sharing of information and digital solutions. We at the BDA have long called for universal access to summary care records and electronic prescribing. Summary Care Record access could be extremely valuable in allowing us to view medical histories, medications and safeguarding alerts and could be of significant benefit to our patients, as well as reducing the associated administrative burden.

Finally, new opportunities and ways of working may allow us to consider how we could support prevention in the future. Some aspects of Delivering Better Oral Health could be delivered remotely and virtual prevention advice and oral health promotion could be considered. High fluoride toothpaste prescriptions have continued to be issued, to support prevention for our patients at high risk of developing caries. With the potential for reduced capacity in dental services and at GA, the prevention agenda must be a priority.

Our community dental teams have risen to these challenges and I am sure that they will continue to do so. Times ahead may be difficult, but I know we will collaborate, learn and share as we go, ensuring that our services adapt to the changes that we will encounter.

\footnotetext{
Reference

1. Westgarth D. COVID-19 and Community Dental Services: The challenges ahead. BDJ In Pract 2020; 33: 14-19.
} 